\title{
Resurrection of PARP Inhibitors in Breast Cancer
}

\author{
Tomas G. Lyons, MD, and Mark E. Robson, MDa,b
}

\section{Abstract}

PARP enzymes are essential for DNA damage repair. Cancers with defective homologous recombination DNA repair, such has BRCA1- and BRCA2-mutated breast cancers, are targets for PARP inhibitors (PARPi) through the exploitation of synthetic lethality. A number of PARPi are currently undergoing clinical evaluation in breast cancer, with olaparib and talazoparib having demonstrated superior efficacy compared with standard chemotherapy in advanced germline BRCA-mutated cancer. This review describes the biological rationale for PARPi and presents the accumulating data on PARPi use in breast cancer.

\section{Role of PARP in DNA Damage Repair}

Cellular function is dependent on the maintenance of genomic integrity. Endogenous and exogenous insults may induce damage to DNA and result in the formation of single-strand DNA breaks (SSBs)., ${ }^{1,2}$ PARP enzymes 1 and 2 are key to the repair of these SSBs. PARP1 binds to the SSBs, which activates its catalytic function and results in PARylation of PARP1. This in turn stimulates the recruitment of DNA repair effectors to the site of DNA damage. AutoPARylation of PARP1 releases PARP1 from the repaired DNA, and cell cycle replication recommences. ${ }^{3}$

If PARP activity is inhibited, the enzyme appears to be physically "trapped" on the DNA, physically obstructing the progress of replication and introducing significant replication stress. Replication fork stalling occurs with subsequent collapse and the formation of double-strand breaks (DSBs). ${ }^{4}$ Homologous recombination-directed repair is a highly accurate process for repairing DSBs. Both BRCA1 and BRCA2 are critical to the process of homologous recombination-directed DNA repair. If homologous recombination repair is impaired by the loss of BRCA1 or BRCA2 function, then

aBreast Medicine Service, and ${ }^{b}$ Clinical Genetics Service, Memorial Sloan Kettering Cancer Center, New York, New York.

Submitted November 18, 2017; accepted for publication April 4, 2018.

Dr. Robson has disclosed that he is a consultant for AstraZeneca and McKesson; receives honoraria from AstraZeneca; and receives grant/ research support from AstraZeneca, AbbVie, Invitae, Medivation, and other DNA repair pathways may be engaged, such as nonhomologous end joining (NHEJ). NHEJ is less accurate, leading to genomic instability, which may be sufficient to result in cell death (Figure 1).

In classical genetics, synthetic lethality describes a situation whereby a defect in either of 2 genes has a limited effect on a cell, but defects in both genes leads to cell death. In 2005, Bryant et $\mathrm{al}^{5}$ and Farmer et $\mathrm{al}^{6}$ demonstrated synthetic lethality proof-of-concept with the PARP inhibitor (PARPi) olaparib in BRCA-mutated cells. ${ }^{5,6}$ This seminal preclinical work paved the way for the subsequent clinical studies of PARPi in homologous recombination-deficient cancers, particularly those arising in BRCA mutation carriers.

Although this model is used to explain the mechanism of PARPi cytotoxicity in the setting of germline BRCA (gBRCA)mutations, PARP1 appears to be involved in other aspects of DNA repair, including repair of DSBs. ${ }^{7}$ Inhibition of these functions may also contribute to cell death. In addition, PARPi has been shown to induce mitophagy (degradation of mitochondria by autophagy) and cell apoptosis. ${ }^{8}$

Myriad Genetics. Dr. Lyons has disclosed that he has no financial interests, arrangements, affiliations, or commercial interests with the manufacturers of any products discussed in this article or their competitors.

Correspondence: Mark E. Robson, MD, Department of Medicine, Memorial Sloan Kettering Cancer Center, 1275 York Avenue, New York, NY 10065. Email: robsonm@mskcc.org 


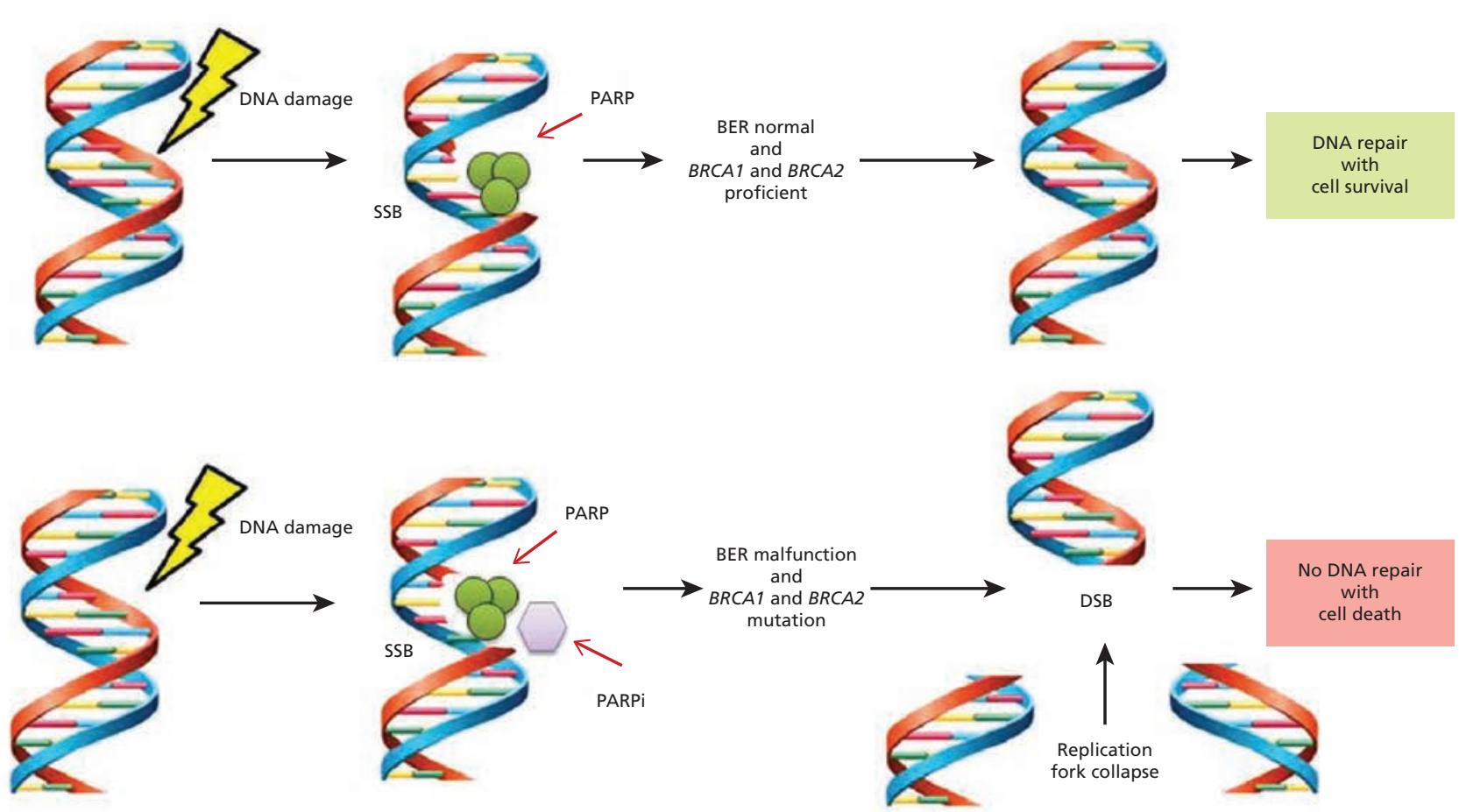

Figure 1. Mechanism of action of PARP inhibition.

Abbreviations: BER, base excision repair; DSB, double strand break; PARPi, PARP inhibitor; SSB, single strand break.

\section{Evolution of PARP Targeting in Breast Cancer}

There are currently a number of PARPi in clinical development, including olaparib, veliparib, niraparib, rucaparib, and talazoparib. These PARPi are similar in that they all inhibit PARP1 and PARP2 catalytic activity but have differing potency in PARP trapping. ${ }^{4}$ Greater PARP trapping has been associated with enhanced preclinical efficacy, but no clinical studies have directly compared different PARPi in patients. ${ }^{9}$

Three PARPi agents are currently FDA-approved for use in ovarian cancer (olaparib, niraparib, and rucaparib), and on January 12, 2018, olaparib received FDA approval for use in patients with gBRCA-mutated, HER2-negative, advanced breast cancer previously treated with chemotherapy and/ or endocrine therapy. Several trials are also studying the benefit of PARPi in other diseases, including prostate and pancreas cancers (Figure 2, Table 1). To date, there have been 2 general therapeutic approaches when targeting PARP in breast cancer: patients with a gBRCA mutation and unselected patients with triple-negative breast cancer (TNBC). The following sections will discuss the most relevant data with respect to the evolution of PARPi for the treatment of advanced or metastatic breast cancer.

\section{Olaparib}

The first-in-human study of olaparib monotherapy in patients with solid tumors identified the maximum tolerated dose to be $400 \mathrm{mg}$ twice daily. ${ }^{10}$ In this study, 19 evaluable patients with gBRCA1/2 mutations were enrolled in an expansion cohort, and $47 \%$ ( 8 ovarian and 1 breast cancer) achieved a complete or partial response. Tutt et al ${ }^{11}$ subsequently conducted a proof-of-concept phase II study in 54 patients with breast cancer with gBRCA mutations, which described an objective response rate (ORR) of $41 \%$ (95\% CI, 25-59) in 27 patients receiving olaparib capsules at $400 \mathrm{mg}$ twice daily and 22\% (95\% CI, 11-41) in patients receiving $100 \mathrm{mg}$ twice daily. Another phase II study by Kaufman et $\mathrm{al}^{12}$ described an ORR of $12.9 \%$ (95\% CI, 5.7-23.9) in 62 heavily pretreated patients with gBRCA-mutated breast cancer, further supporting the clinical activity of the compound.

Most breast cancers in BRCA1 mutation carriers are hormone receptor-negative and do not overexpress HER2 (ie, TNBC). Because of this, there was early hope that TNBC in women without BRCA mu- 


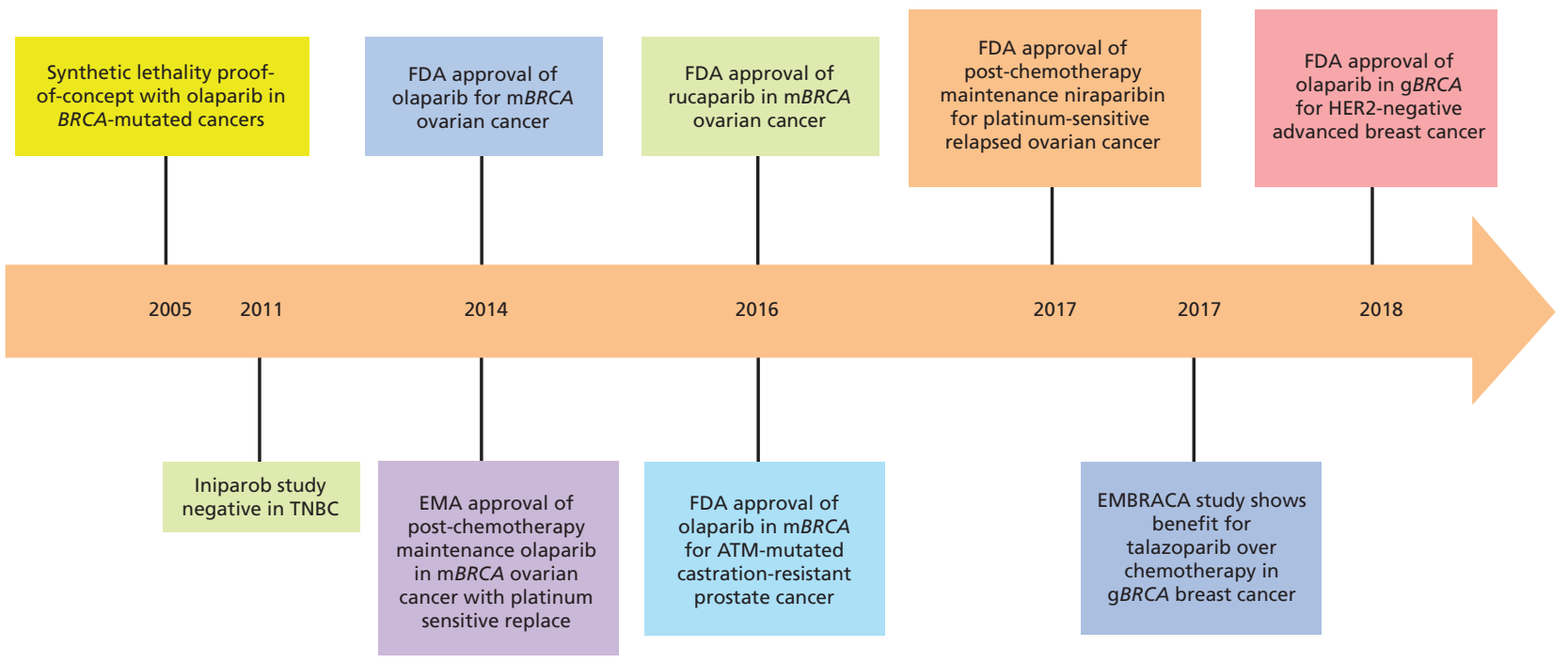

Figure 2. Timeline of PARP inhibitor clinical milestones.

Abbreviations: ATM, ataxia-telangiectasia mutated; EMA, European Medicines Agency; gBRCA, germline $B R C A$; mBRCA, $B R C A$ mutated; TNBC, triple-negative breast cancer.

tations would also harbor defects in homologous recombination that would render it sensitive to PARPi in a manner similar to that observed in BRCA-deficient cells. However, Gelmon et $\mathrm{al}^{13}$ conducted a small phase II study of olaparib in unselected TNBC that casted doubt on this hypothesis. In this study, 23 patients with unselected TNBC were enrolled: 15 were BRCA wild-type and 8 had a gBRCA mutation. The primary end point was ORR, with no confirmed responses in 15 patients with TNBC but without BRCA mutations.
A positive phase II randomized study of another purported PARPi, iniparib, in combination with gemcitabine and carboplatin in patients with metastatic TNBC unselected for BRCA mutations ${ }^{14}$ led to a strategic reevaluation of the gBRCA-directed approach for compounds other than iniparib and to a temporary pause in development in BRCA-mutated breast cancer pending the results of a confirmatory phase III study, which failed to meet its primary end point. ${ }^{15}$ Indeed, 2 groups showed that iniparib failed to inhibit PARP. ${ }^{16,17}$ PARPi development in breast cancer stalled and focus turned toward development

\section{Table 1. PARP Inhibitors in Phase III Trials}

\begin{tabular}{|c|c|c|c|c|c|}
\hline Drug & Trial Identifier & Setting & Design & Population & Status \\
\hline \multirow[t]{2}{*}{ Olaparib } & $\begin{array}{l}\text { OlympiAD } 20 \\
\text { NCT02000622 }\end{array}$ & Advanced/Metastatic & $\begin{array}{l}\text { Olaparib } \\
\text { vs } \\
\text { PCT }\end{array}$ & Advanced/Metastatic gBRCA, $\leq 2$ prior lines & Resulted \\
\hline & $\begin{array}{l}\text { OlympiA } \\
\text { NCT02032823 }\end{array}$ & Adjuvant & $\begin{array}{l}\text { Olaparib } \\
\text { vs } \\
\text { placebo }\end{array}$ & $\begin{array}{l}\text { Early-stage gBRCA, post completion SOC } \\
\text { adjuvant therapy }\end{array}$ & Recruiting \\
\hline \multirow[t]{2}{*}{ Veliparib } & $\begin{array}{l}\text { BROCADE } 3 \\
\text { NCT02163694 }\end{array}$ & Advanced/Metastatic & $\begin{array}{l}\mathrm{C}+\mathrm{P}+\text { veliparib } \\
\text { vs } \\
\mathrm{C}+\mathrm{P}+\text { placebo }\end{array}$ & Metastatic $\mathrm{g} B R C A, 0-2$ lines of prior therapy & Recruiting \\
\hline & $\begin{array}{l}\text { BrighTNess }^{29} \\
\text { NCT02032277 }\end{array}$ & Neoadjuvant & $\begin{array}{l}C+P+\text { veliparib } \rightarrow A C \\
\text { vs } \\
C+P+\text { placebo } \rightarrow A C \\
\text { vs } \\
\text { Placebo }+ \text { placebo }+P \rightarrow A C\end{array}$ & Neoadjuvant TNBC & Resulted \\
\hline Talazoparib & $\begin{array}{l}\text { EMBRACA }^{23} \\
\text { NCT01945775 }\end{array}$ & Advanced/Metastatic & $\begin{array}{l}\text { Talazoparib } \\
\text { vs } \\
\text { PCT }\end{array}$ & Advanced/Metastatic gBRCA, $\leq 3$ prior lines & Resulted \\
\hline Niraparib & $\begin{array}{l}\text { BRAVO } \\
\text { NCT01905592 }\end{array}$ & Advanced/Metastatic & $\begin{array}{l}\text { Niraparib } \\
\text { vs } \\
\text { PCT }\end{array}$ & Advanced/Metastatic gBRCA, $\leq 2$ prior lines & Not recruiting \\
\hline
\end{tabular}

Abbreviations: AC, doxorubicin + cyclophosphamide; C, carboplatin; gBRCA, germline BRCA; P, paclitaxel; PCT, physician's choice chemotherapy; SOC, standard of care; TNBC, triple-negative breast cancer. 
in ovarian cancer. Fortunately, the benefits of PARPi in BRCA-deficient ovarian cancers were sufficient to rekindle interest in the breast cancer arena, ${ }^{18,19}$ and the phase III OlympiAD study of olaparib compared with physician's choice of chemotherapy (PCT) was launched in patients with BRCA mutations and metastatic HER2 non-overexpressing breast cancer. ${ }^{20}$

OlympiAD was a randomized, open-label, phase III trial evaluating olaparib monotherapy (300-mg tablets twice daily) compared with conventional chemotherapy. ${ }^{20}$ A total of 302 patients who had received $\leq 2$ prior therapies in the advanced setting were randomized 2:1 to the study arms. After a median follow-up of 14.5 months, progression-free survival (PFS), the primary end point, was significantly prolonged with olaparib versus standard therapy ( 7.0 vs 4.2 months; hazard ratio [HR], 0.58; $95 \%$ CI, $0.43-0.8 ; P<.001)$; response rate in the olaparib group was also increased $(59.9 \%$ vs $28.8 \%)$. At planned interim analysis, there was no difference in overall survival between the 2 groups. No new safety signals were observed. Any-grade nausea and vomiting were increased with olaparib compared with standard chemotherapy ( $58 \%$ vs $35.2 \%$, respectively, and $29.8 \%$ vs $15.4 \%$, respectively). Olaparib was associated with fewer overall grade $3 / 4$ toxicities, with anemia the only grade $3 / 4$ adverse event seen more frequently in this group ( $16.1 \%$ vs $4.4 \%)$. Olaparib was the first PARPi to demonstrate superior efficacy and better tolerability compared with standard chemotherapy in gBRCA-mutated advanced breast cancer, resulting in its FDA approval for treatment of this patient subgroup.

\section{Talazoparib}

Talazoparib is another PARPi undergoing evaluation in breast cancer. A phase I trial in patients with gBRCA-mutated breast cancer determined that 1 $\mathrm{mg} / \mathrm{d}$ was the recommended phase II dose. ${ }^{21}$ ORR in patients with breast cancer was 50\%, with 7 of 14 patients experiencing a response ( 1 complete and 6 partial responses). The clinical benefit rate at $\geq 24$ weeks was $85.7 \%$. Additionally, results of the phase II ABRAZO trial have been presented in abstract form. ${ }^{20}$ The 2 cohorts in this study included patients with gBRCA-mutated breast cancer with previous exposure to platinum (cohort 1) and those with no prior platinum exposure but who received $\geq 3$ lines of therapy (cohort 2). The ORR was $21 \%$ in cohort
1 and 37\% in cohort 2, and the ORRs by BRCA mutation and subtype of breast cancer were $24 \%$ for BRCA1, 34\% for BRCA2, and 26\% for TNBC. The most frequently reported adverse events were anemia (52\%), fatigue (45\%), and nausea (42\%).

The EMBRACA study is evaluating talazoparib as monotherapy in patients with gBRCA-mutated breast cancer compared with PCT, with a similar design to the OlympiAD trial. In this open-label phase III study, 287 patients were randomized to talazoparib at $1 \mathrm{mg}$ and 144 patients to PCT. ${ }^{23}$ All patients had gBRCA-mutated, HER2-negative, advanced breast cancer and could have received no more than 3 prior lines of chemotherapy in the advanced setting. Median PFS, which was the primary end point, was significantly improved with talazoparib compared with PCT (8.6 vs 5.6 months; HR, 0.542; P<.0001), and the ORR was superior $(62.6 \%$ vs $27.2 \%$; HR, 4.99 ; $P<.0001)$. An interim analysis of overall survival seemed to show a positive trend in favor of talazoparib, although these data are immature. Any hematologic adverse event of grade 3 or 4 were more frequent in patients treated with talazoparib versus PCT $(54.9 \%$ vs $38.1 \%)$, including grade 3 or 4 anemia (39.2\% vs $4.8 \%)$ and thrombocytopenia (14.7\% vs $1.6 \%)$; however, grade 3 or 4 neutropenia was less frequent with talazoparib (20.9\% vs $34.9 \%)$.

\section{Veliparib}

Veliparib has been evaluated as both a monotherapy and in combination with chemotherapy in patients with breast cancer. Phase I studies in TNBC established $400 \mathrm{mg}$ twice daily as the monotherapy dose, with an ORR of $60 \%$ in patients with gBRCAmutated breast cancer versus $5 \%$ in those with wildtype disease. ${ }^{24}$ A subsequent phase II study of singleagent veliparib showed a PFS of 5.2 months, with a response rate of $14 \%$ and $36 \%$ for patients with BRCA1 and BRCA2 mutations, respectively. ${ }^{25} \mathrm{Re}$ sults of combining veliparib with carboplatin and paclitaxel in the randomized phase II BROCADE study in patients with gBRCA-mutated breast cancer have been published in abstract form. ${ }^{26}$ The combination resulted in a significantly improved ORR compared with carboplatin/paclitaxel and placebo $(77.8 \%$ vs $61.3 \% ; P=.027)$. PFS was 14.1 months for the veliparib arm versus 12.3 months for placebo, which was not statistically significant. No increase in toxicity was reported in either arm. A confirmatory phase III 
Lyons and Robson

BROCADE 3 study is currently ongoing (ClinicalTrials.gov identifier: NCT02163694).

\section{Niraparib}

Other PARPi have been more difficult to combine with cytotoxic chemotherapy due to myelosuppression, and development has been proceeding largely as single agents in gBRCA breast cancer. Niraparib was recently approved for use as postchemotherapy maintenance in patients with platinum-sensitive relapsed ovarian cancer. ${ }^{27}$ The open-label randomized phase III BRAVO trial (ClinicalTrials.gov identifier: NCT01905592) was evaluating niraparib compared with PCT in patients with gBRCA-mutated breast cancer in a similar design to the OlympiAD study, but was closed early and has not been reported.

\section{PARPi in Early-Stage Breast Cancer}

The long established paradigm in breast cancer has been the transition of a therapy to the adjuvant setting once it has been proven to be both tolerable and efficacious in advanced disease. The transition of PARPi has been no different, with a number of agents being evaluated at an earlier disease stage.

OlympiA is a phase III randomized study evaluating olaparib at $300 \mathrm{mg}$ twice daily for 1 year in patients with gBRCA1/2 mutation with residual disease after neoadjuvant chemotherapy; those with node-positive TNBC or node-negative TNBC with a tumor measuring $\geq 2 \mathrm{~cm}$ following adjuvant chemotherapy; or those with estrogen receptor-positive disease with $\geq 4$ nodes following surgery and adjuvant chemotherapy (ClinicalTrials.gov identifier: NCT02032823).

Veliparib was evaluated in patients with TNBC as part of the I-SPY 2 study. ${ }^{28}$ Patients received weekly paclitaxel with or without carboplatin and veliparib, followed by standard doxorubicin and cyclophosphamide in the neoadjuvant setting. The predicted pathologic complete response (pCR) rate was $51 \%$ versus $26 \%$ in the control arm. These results revealed an $88 \%$ projected probability of success in a phase III study, leading to graduation of this combination from the I-SPY 2 platform. However, results of the 3-arm, randomized, placebo-controlled phase III BrighTNess study (ClinicalTrials.gov identifier: NCT02032277), which evaluated the addition of veliparib to carboplatin versus carboplatin alone versus placebo followed by standard chemotherapy given neoadjuvantly in patients with TNBC, failed to show an improvement in pCR for the combination of veliparib and carboplatin versus carboplatin alone $\left(53.2 \%\right.$ vs $57.5 \%$, respectively). ${ }^{29}$

Lastly, talazoparib is being tested as monotherapy for a 4- to 6-month duration in the neoadjuvant setting for patients with a gBRCA mutation (ClinicalTrials.gov identifier: NCT02282345). A pilot study by the same group investigated single-agent talazoparib for 2 months in a similar patient cohort, followed by standard chemotherapy, and results showed that all 13 patients enrolled had a decrease in tumor volume, with an average reduction of $78 \%$ (range, $30 \%-98 \%) .{ }^{30}$

\section{Combining PARPi With Other Therapies}

The strategy for combining PARPi with other therapeutic agents aims to increase response rates, prevent or delay resistance, and improve clinical outcomes. Cytotoxic chemotherapy is the obvious companion to pair with a PARPi, and numerous studies with differing agents have attempted this strategy but have been limited by toxicity, especially myelosuppression. ${ }^{31-33}$ Approaches to overcoming the toxicity have included reducing the PARPi dose, abbreviating the PARPi dosing schedule, or using a lower dose of the chemotherapeutic agent (ClinicalTrials.gov identifiers: NCT02561832, NCT02264678, NCT02358200). However, whether any of these strategies will lead to improved outcomes compared with using maximal doses of either agent is unclear.

Combining immunotherapy with PARPi is a promising approach with limited expected cross-toxicity. Sound clinical rationale exists for combining a checkpoint inhibitor with PARPi in BRCA-mutated cancers, because these cancers are more genomically unstable due to the defect in homologous recombination, and therefore may be more immunogenic. Data show that tumors with a defect in mismatch repair, which is another DNA repair pathway, are responsive to the PD-1 inhibitor pembrolizumab. ${ }^{34}$ As of May 2017, pembrolizumab is FDA-approved for adults and pediatric patients with advanced mismatch repair-deficient solid tumors that have progressed on prior therapy.

The MEDIOLA trial is a phase I/II open-label basket study of olaparib and durvalumab in patients 
with advanced solid tumors. ${ }^{35}$ Results were recently presented for the cohort with HER2-negative and gBRCA mutation-positive advanced breast cancer. Patients could not have received a PARPi or immunotherapy, prior anthracycline and taxane was required, and prior platinum therapy was allowed. $\mathrm{Pa}$ tients received single-agent olaparib, $300 \mathrm{mg}$ orally twice daily for 4 weeks, after which durvalumab, 1.5 g intravenously was added every 4 weeks. A total of 25 patients were enrolled, 12 (48\%) having estrogen receptor-positive disease and 13 (52\%) having TNBC. The ORR was $67 \%$ in patients with no prior therapy ( 6 of 9 ), $67 \%$ in patients with 1 prior therapy (6 of 9 ), $20 \%$ in patients with 2 prior therapies ( 1 of 5 ), and $0 \%$ in patients with $\geq 3$ prior therapies ( 0 of 2). Median PFS had not been reached, with data cutoff at 6 months. The combination was generally well tolerated, with no unexpected toxicity observed. Grade $\geq 3$ events included anemia (8\%), neutropenia $(8 \%)$, and fatigue $(4 \%)$, and were all attributed to olaparib. A single case of grade $\geq 3$ of both hemolysis and pancreatitis was reported and thought to be related to durvalumab therapy.

Niraparib is also being investigated in combination with the PD-1 inhibitor pembrolizumab in a phase I/II study in TNBC and ovarian cancer (ClinicalTrials.gov identifier: NCT02657889). Additional immunotherapy and PARPi combination studies are also in development.

Early-phase studies are investigating the combination of PARPi with inhibitors of cell cycle checkpoints and DNA repair. The biological rationale for combining PARPi with other compounds (eg, ATR, WEE1, and CHEK1/2 inhibitors) that also inhibit DNA repair pathways is that by inhibiting 2 repair pathways simultaneously, cancer cells have fewer ways to repair damaged DNA, leading to apoptosis. These approaches are being evaluated in a number of different cancer types, including breast cancer. For example, the ATR inhibitor AZD6738 is being evaluated in a phase I study in combination with olaparib in patients with breast cancer with a germline or somatic BRCA mutation. This study also includes a cohort of patients with non-BRCA-mutated TNBC (ClinicalTrials.gov identifier: NCT02264678). An openlabel, randomized, multicenter phase II study will assess the efficacy and safety of olaparib monotherapy versus olaparib in combination with AZD6738 and in combination with the WEE1 inhibitor AZD1775 in patients with TNBC (NCT03330847). And the CHEK1/2 inhibitor (LY2606368) is being investigated in a phase II study in BRCA1/2 mutation-associated breast cancer, TNBC, ovarian cancer, and prostate cancer (NCT02203513).

\section{Conclusions}

PARP is now a proven target, with its inhibition showing significant clinical benefit in a phase III study in patients with gBRCA mutation-positive breast cancer. However, the road traveled by PARPi has not been without its obstacles. Despite early signals of activity of PARPi in breast cancer, the failure of iniparib interrupted further development of these drugs for this cancer type. Fortunately, compelling clinical data, particularly in ovarian cancer, led to a recommitment to the evaluation of these agents. Olaparib was the first PARPi to demonstrate superior efficacy compared with standard chemotherapy in patients with gBRCA-mutated advanced breast cancer, resulting in its regulatory approval. This milestone marks the end of the beginning for PARP targeting in breast cancer. Ongoing and future studies are required to better define which patients to treat with PARPi and whether the target population can be extended beyond those with gBRCA mutations. It will also be important to assess when to treat these patients; how to sequence these drugs with currently established therapies, particularly platinum agents; how to combine PARPi with other novel therapies; and how to overcome resistance. The hope is that these trials will incorporate informative correlative components that will further our understanding of the biology of targeting PARP in breast cancer and add to the arsenal of therapies available, ultimately improving patient outcomes.

\section{References}

1. Hoeijmakers JH. Genome maintenance mechanisms for preventing cancer. Nature 2001;411:366-374.

2. Hoeijmakers JH. DNA damage, aging, and cancer. N Engl J Med 2009;361:1475-1485.

3. Lord CJ, Ashworth A. PARP inhibitors: synthetic lethality in the clinic.
Science 2017;355:1152-1158

4. Pommier Y, O'Connor MJ, de Bono J. Laying a trap to kill cancer cells: PARP inhibitors and their mechanisms of action. Sci Transl Med 2016;8:362ps17.

5. Bryant HE, Schultz N, Thomas HD, et al. Specific killing of BRCA2deficient tumours with inhibitors of poly(ADP-ribose) polymerase. Nature 2005;434:913-917. 
Lyons and Robson

6. Farmer H, McCabe N, Lord CJ, et al. Targeting the DNA repair defect in BRCA mutant cells as a therapeutic strategy. Nature 2005;434:917-921.

7. De Vos M, Schreiber V, Dantzer F. The diverse roles and clinical relevance of PARPs in DNA damage repair: current state of the art. Biochem Pharmacol 2012;84:137-146.

8. Arun B, Akar U, Gutierrez-Barrera AM, et al. The PARP inhibitor AZD2281 (Olaparib) induces autophagy/mitophagy in BRCA1 and BRCA2 mutant breast cancer cells. Int J Oncol 2015;47:262-268.

9. Murai J, Pommier Y. Classification of PARP inhibitors based on PARP trapping and catalytic inhibition, and rationale for combinations with topoisomerase I inhibitors and alkylating agents. In: Curtin N, Sharma R, eds. PARP Inhibitors for Cancer Therapy. New York, NY: Humana Press; 2015:261-274.

10. Fong PC, Boss DS, Yap TA, et al. Inhibition of poly(ADP-ribose) polymerase in tumors from BRCA mutation carriers. N Engl J Med 2009;361:123-134.

11. Tutt A, Robson M, Garber JE, et al. Oral poly(ADP-ribose) polymerase inhibitor olaparib in patients with BRCA1 or BRCA2 mutations and advanced breast cancer: a proof-of-concept trial. Lancet 2010;376:235-244.

12. Kaufman B, Shapira-Frommer R, Schmutzler RK, et al. Olaparib monotherapy in patients with advanced cancer and a germline BRCA1/2 mutation. J Clin Oncol 2015;33:244-250.

13. Gelmon KA, Tischkowitz M, Mackay $\mathrm{H}$, et al. Olaparib in patients with recurrent high-grade serous or poorly differentiated ovarian carcinoma or triple-negative breast cancer: a phase 2, multicentre, open-label, nonrandomised study. Lancet Oncol 2011;12:852-861.

14. O'Shaughnessy J, Osborne C, Pippen JE, et al. Iniparib plus chemotherapy in metastatic triple-negative breast cancer. N Engl J Med 2011;364:205-214.

15. O'Shaughnessy J, Schwartzberg L, Danso MA, et al. Phase III study of iniparib plus gemcitabine and carboplatin versus gemcitabine and carboplatin in patients with metastatic triple-negative breast cancer. J Clin Oncol 2014;32:3840-3847.

16. Patel AG, De Lorenzo SB, Flatten KS, et al. Failure of iniparib to inhibit poly(ADP-Ribose) polymerase in vitro. Clin Cancer Res 2012;18:16551662.

17. Liu X, Shi Y, Maag DX, et al. Iniparib nonselectively modifies cysteinecontaining proteins in tumor cells and is not a bona fide PARP inhibitor. Clin Cancer Res 2012;18:510-523

18. Kaye SB, Lubinski J, Matulonis U, et al. Phase II, open-label, randomized, multicenter study comparing the efficacy and safety of olaparib, a poly (ADP-ribose) polymerase inhibitor, and pegylated liposomal doxorubicin in patients with BRCA1 or BRCA2 mutations and recurrent ovarian cancer. J Clin Oncol 2012;30:372-379.

19. Ledermann J, Harter P, Gourley C, et al. Olaparib maintenance therapy in platinum-sensitive relapsed ovarian cancer. N Engl J Med 2012;366:1382 1392.

20. Robson M, Im SA, Senkus E, et al. Olaparib for metastatic breast cancer in patients with a germline BRCA mutation. N Engl J Med 2017;377:523-533.

21. de Bono J, Ramanathan RK, Mina L, et al. Phase I, dose-escalation, twopart trial of the PARP Inhibitor talazoparib in patients with advanced germline BRCA1/2 mutations and selected sporadic cancers. Cancer Discov 2017;7:620-629.

22. Turner NC, Telli ML, Rugo HS, et al. Final results of a phase 2 study of talazoparib (TALA) following platinum or multiple cytotoxic regimens in advanced breast cancer patients (pts) with germline BRCA1/2 mutations (ABRAZO) [abstract]. J Clin Oncol 2017;35(15 Suppl):Abstract 1007.

23. Litton J, Rugo HS, Ettl J, et al. EMBRACA: a phase 3 trial comparing talazoparib, an oral PARP inhibitor, to physician's choice of therapy in patients with advanced breast cancer and a germline BRCA mutation. Presented at the 2017 San Antonio Breast Cancer Symposium; December 5-9, 2017; San Antonio, Texas. Abstract GS6-07.

24. Puhalla $S$, Beumer JH, Pahuja $S$, et al. Final results of a phase 1 study of single-agent veliparib $(\mathrm{V})$ in patients (pts) with either BRCA1/2-mutated cancer (BRCA + ), platinum-refractory ovarian, or basal-like breast cancer (BRCA-wt) [abstract]. J Clin Oncol 2014;32(Suppl):Abstract 2570.

25. Somlo G, Frankel PH, Arun BK, et al. Efficacy of the PARP inhibitor veliparib with carboplatin or as a single agent in patients with germline BRCA1 - or BRCA2-associated metastatic breast cancer: California Cancer Consortium Trial NCT01149083. Clin Cancer Res 2017;23:4066-4076.

26. Han HS, Sook DV, Robson ME, et al. Efficacy and tolerability of veliparib (V; ABT-888) in combination with carboplatin (C) and paclitaxel (P) vs placebo $(\mathrm{Plc})+\mathrm{C} / \mathrm{P}$ in patients (pts) with BRCA1 or BRCA2 mutations and metastatic breast cancer: a randomized, phase 2 study [abstract]. Presented at the 2016 San Antonio Breast Cancer Symposium; December 6-10, 2016; San Antonio, Texas. Abstract S2-05

27. Mirza MR, Monk BJ, Herrstedt J, et al. Niraparib maintenance therapy in platinum-sensitive, recurrent ovarian cancer. N Engl J Med 2016;375:21542164.

28. Rugo HS, Olopade OI, DeMichele A, et al. Adaptive randomization of veliparib-carboplatin treatment in breast cancer. $N$ Engl J Med 2016;375:23-34.

29. Loibl S, O'Shaughnessy J, Untch M, et al. Addition of the PARP inhibitor veliparib plus carboplatin or carboplatin alone to standard neoadjuvant chemotherapy in triple-negative breast cancer (BrighTNess): a randomised phase 3 trial. Lancet Oncol 2018;19:497-509.

30. Litton JK, Scoggins M, Ramirez DL, et al. A feasibility study of neoadjuvant talazoparib for operable breast cancer patients with a germline BRCA mutation demonstrates marked activity. NPJ Breast Cancer 2017;3:49.

31. Khan $\mathrm{OA}$, Gore M, Lorigan $\mathrm{P}$, et al. A phase I study of the safety and tolerability of olaparib (AZD2281, KU0059436) and dacarbazine in patients with advanced solid tumours. Br J Cancer 2011;104:750-755.

32. Rajan A, Carter CA, Kelly RJ, et al. A phase I combination study of olaparib with cisplatin and gemcitabine in adults with solid tumors. Clin Cancer Res 2012;18:2344-2351

33. Balmana J, Tung NM, Isakoff SJ, et al. Phase I trial of olaparib in combination with cisplatin for the treatment of patients with advanced breast, ovarian and other solid tumors. Ann Oncol 2014;25:1656-1663.

34. Le DT, Uram JN, Wang H, et al. PD-1 blockade in tumors with mismatchrepair deficiency. N Engl J Med 2015;372:2509-2520.

35. Domchek SM, Postel-Vinay S, Bang YJ, et al. An open-label, multitumor, phase II basket study of olaparib and durvalumab (MEDIOLA): results in germline BRCA-mutated (gBRCAm) HER2-negative metastatic breast cancer (MBC) [abstract]. Presented at the San Antonio Breast Cance Symposium; December 5-9, 2017; San Antonio, Texas. Abstract PD6-11. 